PRAXIS

ecucativa

Universidad Nacional de La Pampa

Facultad de Ciencias Humanas

Instituto de Ciencias de la Educación para la investigación interdisciplinaria

\section{†

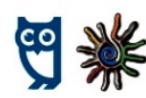 \\ Instituto de Ciencias de la Educación
para la Investigación Interdisciplinarlit

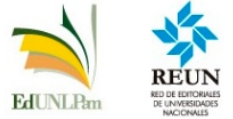

ISSN 2313-934X

SANTA ROSA, LA PAMPA, ARGENTINA

Correo electrónico: iceii@humanas.unlpam.edu.ar

Disponible en https://cerac.unlpam.edu.ar/index.php/praxis

Categorías de análisis en la planificación de la intervención territorial en el marco de las prácticas integrales. Artículo de Carlos Alberto Zavaro Pérez. Praxis educativa, Vol. 25, No 3 septiembre - diciembre 2021. E - ISSN 2313-934X. pp. 1-22. https://dx.doi.org/10.19137/praxiseducativa-2021-250307

Esta obra se publica baja Licencia Creative Commons 4.0 Internacional CC BY-NC-SA Atribución, No Comercial, Compartir igual

(c) (1) $\$$

\title{
Categorías de análisis en la planificación de la intervención territorial en el marco de las prácticas integrales
}

Categories of analysis in the planning of territorial intervention within the framework of comprehensive practices

Categorias de análise no planejamento da intervenção territorial no quadro de práticas integrais

\section{Carlos Alberto Zavaro Pérez}

Universidad Nacional de La Plata

Universidad de Belgrano

Argentina

czavaro@fcnym.unlp.edu.ar

ORCID 0000-0003-3298-7383

Recibido: 2021-01-01 | Revisado: 2021-04-03 | Aceptado: 2021-05-05 


\section{Resumen}

Las prácticas integrales constituyen instancias de formación de grado y de articulación con la comunidad. En ese marco, es posible integrar las tradiciones de la investigación, de la extensión y la enseñanza desde un paradigma dialógico y constructivista en un espacio pedagógico que, por otra parte, permite abordar los problemas que atraviesan al territorio. El éxito del formato, no obstante, depende de una gestión eficiente, por lo que es importante una planificación que garantice su objetivación y su evaluación permanente y que constituye, además, un aprendizaje. Contexto, problematización, diagnóstico, objetivos, metas, metodología (que incluye actividades, dinámicas y recursos) y herramientas para una planificación y evaluación estratégica situacional constituyen algunas de las categorías que permiten diseñar el formato en que se objetiva la docencia.

Palabras clave: contexto, planificación, metodología, evaluación, prácticas integrales.

\section{Abstract}

Integral practices constitute instances of undergraduate training and articulation with the community. In this framework, it is possible to integrate the traditions of research, extension and teaching from a dialogic and constructivist paradigm in a pedagogical scheme that, in addition, allows addressing the problems that cross the territory. The success of the format depends on efficient management, that is why planning that guarantees its objectification and permanent evaluation is important. Context, problematization, diagnosis, objectives, goals, methodology (which includes activities, dynamics and resources) and tools, for a situational strategic planning and evaluation, constitute some of the categories that allow designing the format in which teaching is objectified.

Keywords: context; design; methodology; evaluation; integral practices.

\section{Resumo}

As práticas integrais constituem instâncias de formação e articulação com a comunidade. Nesse contexto, é possível integrar as tradições de pesquisa, extensão e ensino a partir de um paradigma dialógico e construtivista em um esquema pedagógico que, além disso, permite abordar os problemas que atravessam o território. O sucesso do formato depende de uma gestão eficiente, por isso é importante um planejamento que garanta sua objetivação e avaliação permanente. Contexto, problematização, diagnóstico, objetivos, metas, metodologia (que inclui atividades, dinâmicas e recursos) e ferramentas, para um planejamento e avaliação estratégica situacional, constituem algumas das categorias que permitem desenhar o formato em que se objetiva o ensino.

Palavras-chave: contexto; planejamento; metodologia; avaliação; práticas integrais. 


\section{Introducción}

Las prácticas integrales constituyen un tema en debate que ha ido ocupando paulatinamente la centralidad de la actividad académica y que tiene una enorme relevancia, en particular, cuando esta es concebida como una experiencia pedagógica directamente vinculada con la formación. En ellas, no solo confluyen diversas disciplinas en torno al trabajo sobre la base de problemas del territorio, sino que, en su abordaje, promueven la articulación de metodologías, sujetos y funciones académicas como la docencia, la investigación, la extensión y la gestión. Si la enseñanza de los temas que comprende el programa de estudio de una materia son concebidos con un anclaje territorial y en contacto estrecho con la comunidad, la extensión entonces no solo termina encontrando modos alternativos en los que objetiva su curricularización (Zavaro, 2019), sino también se convierte en el eje que vertebra el formato, tanto por su tradición en la relación que históricamente ha establecido con la comunidad, como por su capacidad de amalgamar y "diluir" a las otras prácticas (Zavaro, 2020a) que tradicionalmente han sido concebidas como disociadas.

Un formato de enseñanza de estas características supone una enorme complejidad y esto representa un desafío adicional por la multiplicidad de variantes y factores que es necesario conjugar si se pretende garantizar una práctica situada que sea relevante y que permita alcanzar los propósitos que la sustentan: la formación contextualizada de futuros profesionales comprometidos con su tiempo y con la sociedad; la articulación sostenida y simbiótica de las universidades con la comunidad en la que se inserta y con sus problemas; y el abordaje dialógico y constructivo de los problemas mismos en la transformación del territorio como resultado de esa intervención.

En ese marco, la gestión de la práctica por parte de los docentes y de los referentes de la institución no solo es esencial como garantía del éxito del formato, sino que constituye una función a integrar, siendo que en esa integración radica uno de los logros colaterales de la experiencia. Incorporar la gestión como aprendizaje y competencia es todo un desafío que promueve la autonomía de quienes participan del espacio, entendiéndolo también como un proceso de aprendizaje colectivo. Esta posibilidad de gestionar colectivamente -cogestionar- implica involucrar diferentes actores y opiniones en el diseño, complejizándolo. Proponer un conjunto de categorías relacionales como estructura arquetípica del formato y como sustento de la reflexión en torno a su objetivación constituye el propósito de estas líneas que, además, pretenden ser útiles en la comprensión y en el diseño de un formato espacio tan singular.

\section{Las categorías}

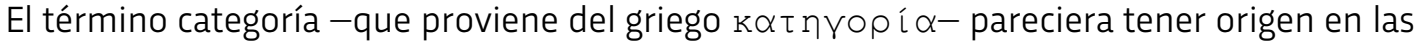
nociones abstractas de Aristóteles y constituyen clases o encuadres con diversos grados de interdependencia, en los que anidan representaciones conceptuales de hechos o de elementos que comparten ciertas regularidades. A partir de ellas, es posible sistematizar y ordenar la reflexión en 
torno a un tema o a un hecho en particular especialmente cuando este resulta complejo y está atravesado por diversos factores que lo definen y condicionan.

Entender y diseñar las prácticas integrales como prácticas situadas y complejas, incorporando a la cogestión (Carpinetti, 2005) como aprendizaje que las transmuta y reconfigura permanentemente, requiere entonces de la definición de algunas categorías que permitan conformar un encuadre o sistema prototípico al que pueda anclarse el diseño de cualquier formato de estas características. Estas categorías constituyen la estructura desde donde es posible vertebrar la práctica y, a la vez, representan instancias de articulación entre las etapas y situaciones que lo configuran (Figura 1).

Un primer bloque referido al escenario en que acontece la práctica incluye categorías como Diagnóstico, Contexto y Problematización que permiten vincular las prácticas de investigación a la práctica docente en pos de un modo de aproximarse al conocimiento del territorio y de sus problemas. Un segundo bloque derivado del conocimiento de este contexto condensa a aquellas que permiten esbozar las razones que sustentan la práctica para lo cual se distinguen las categorías de Fundamentación y Justificación, que a menudo suelen ser confundidas o parecieran ser utilizadas como sinónimo cuando no lo son, así como otro bloque de categorías que, de igual manera, están inevitablemente vinculadas con el diagnóstico sobre el contexto y desde donde pueden ser definidos los propósitos de la intervención que, no obstante, se vinculan con la argumentación de la práctica. Este bloque de categorías se sustenta en la formulación de preguntas, que permiten formular las hipótesis de intervención, definir los objetivos de trabajo y establecer las metas e indicadores que posibiliten dimensionar el impacto de la práctica.

En este sentido, la formulación de objetivos impacta directamente en el diseño de los dispositivos y de las actividades que vertebran el desarrollo de la práctica y está inevitablemente mediada por la utilización de diversas herramientas que, usualmente, son definidas en atención a metodologías particulares que responden a marcos teóricos determinados desde donde es concebida la práctica y en la que se despliegan instrumentos específicos que, en algunos casos, son organizadores de la práctica misma y por tanto de su planificación.

El conjunto de estos instrumentos constituyen, además, insumos de análisis para la evaluación permanente del formato, lo que a su vez contribuye no solo a su adecuación dialéctica, sino también a profundizar, de manera sostenida, en el diagnóstico del contexto y en el modo en que este es atravesado por las intervenciones, relevando el impacto que tiene en la consolidación del espacio y en la formación de quienes lo integran, en especial de las/os estudiantes, en tanto sea concebido como parte de la currícula. 


\section{Figura 1}

Estructura y relaciones en las categorías de análisis y diseño de las prácticas integrales

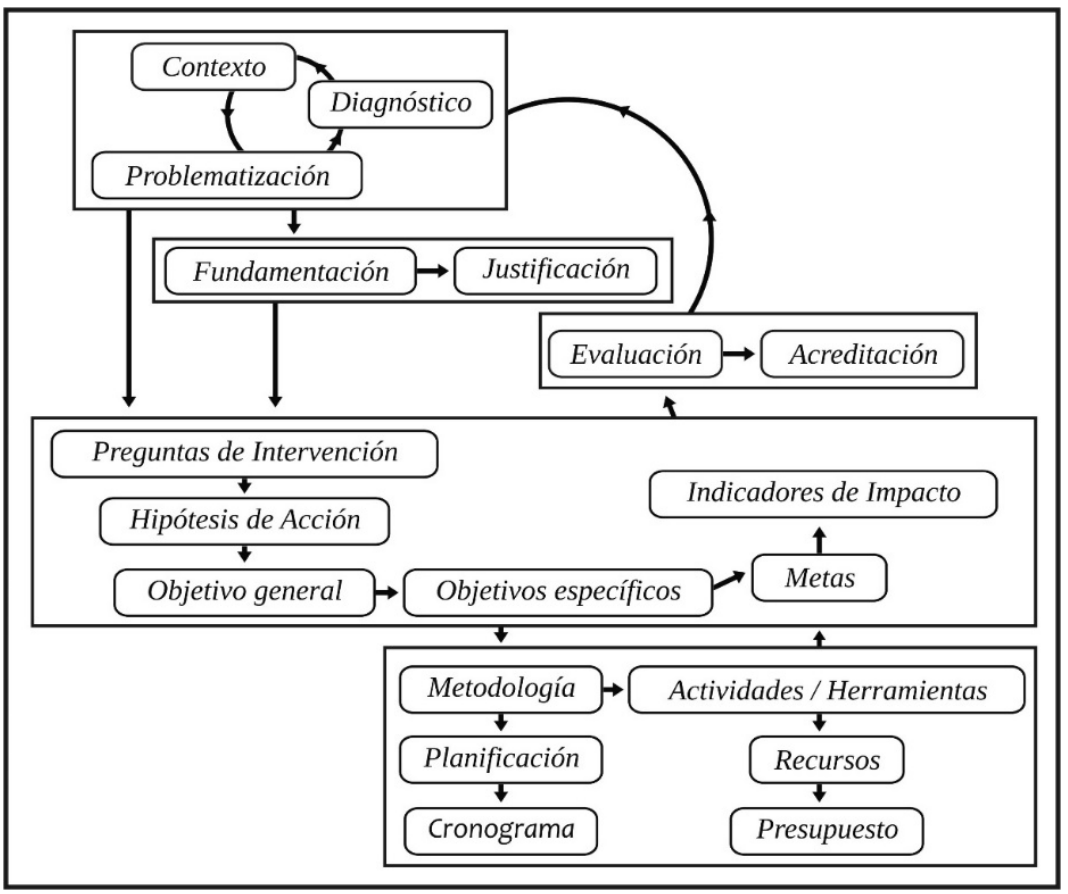

El desarrollo metodológico de una propuesta basada en categorías capaces de articularse entre sí, bajo la lógica de las prácticas integrales, alientan los párrafos que siguen y que, si bien tienen un corte netamente teórico, se sustentan en reflexiones emergentes de prácticas acumuladas por diferentes espacios de esta naturaleza desarrollados en la Universidad Nacional de La Plata.

\section{Contexto y Diagnóstico: la problematización como nexo}

El contexto, en términos socioculturales, nos remite al conjunto de condiciones, de particularidades y circunstancias en que ocurre un hecho concreto: un escenario situado en tiempo y espacio (Rodrigo, 1994) en el que acontece la cotidianidad, pero que no se restringe únicamente a lo externo, sino que además constituye una representación mental de quienes lo conforman y usualmente se encuentran atados a las experiencias cotidianas (van Dijk, 2001). El contexto, en el marco de la extensión universitaria, es el territorio, los barrios que son habitados por vecinas/os que convergen en los clubes, las organizaciones políticas y sociales, en las canchitas de fútbol, las iglesias locales o en espacios culturales que se convierten en sitios de referencia para la comunidad, aun cuando esta pueda estar o no organizada.

El contexto circunda el barrio y lo constituye, lo describe en atención a sus características, a su historia y a los problemas cotidianos que lo atraviesan y que son modulados por el modo en que estos impactan en quienes lo habitan y por la manera en que quienes conviven en esa cotidianidad lo moldean y transforman en función de sus decisiones, de sus imaginarios y de sus 
concepciones del mundo que, en cierta medida, están atravesadas por tensiones y disputas de sentido, pero también por los acuerdos que en esa coyuntura puedan establecerse.

En este escenario, el contexto se configura como una posibilidad para la objetivación de prácticas situadas que, según Ariztía (2017), surgen de la interrelación espacio temporal de tres elementos: competencias, sentido y materialidades, y que, en relación con la perspectiva de la integralidad y de la consecuente integración de funciones académicas, contribuyen al abordaje de los problemas existentes en el territorio, lo que no solo constituye para la universidad un espacio de trabajo y de formación de quienes conforman sus estamentos, que impacta particularmente en la formación de las/os estudiantes y futuros egresadas/os, sino que, además, constituye una oportunidad - para la comunidad- de establecer vínculos sostenidos con la comunidad académica como forma de articulación simbiótica y de construcción de una nueva comunidad de sentido.

El contexto es en sí mismo un espacio para la construcción de un tipo de demanda (Giraudo y Vergerio, 2017) que no se sustenta en los imaginarios concebidos desde los escritorios y las aulas, sino que es resultante de un diagnóstico de necesidades concretas que solo es posible en la medida en que se fomenten y consoliden esos vínculos y se establezcan formas empáticas de aproximarse a la lectura de la realidad que, en principio, es desconocida para quienes, desde la academia, comienzan a intervenir en un territorio particular. Establecer los vínculos necesarios para poder avanzar en el trabajo no es tarea fácil, no siempre las/os vecinas/os pueden estar interesados y mucho menos confían en quienes, hasta ese momento, les resultaban absolutamente desconocidos. En esta empresa, resulta fundamental la articulación temprana con las organizaciones barriales y sus referentes para concretar ese acercamiento sin el cual no es posible abordar los problemas ni definir expectativas de logro y prioridades en su abordaje.

Profundizar en el conocimiento de ese contexto en el que se planifica la intervención requiere del despliegue de diversos modos de encarar el diagnóstico. Esto implica no solo una articulación de actores, sino también de metodologías propias de aquellas disciplinas que intervienen e interactúan. Si bien el diagnóstico no constituye en sí mismo un proyecto de investigación en los términos en que se produce conocimiento científico en la academia, este no está exento de la utilización de las mismas metodologías que la investigación (según la naturaleza del problema a indagar) ni de la rigurosidad que la caracteriza, constituyendo una aproximación que, en el marco de la pedagogía de la integralidad, incorpora conocimientos disciplinares, que en tanto sea capaz de integrar las prácticas de enseñanza-aprendizaje potencia el trabajo extensionista e incluso contribuye a generar otras preguntas que podrían definir, a posteriori, nuevas líneas de investigación.

Diagnosticar implica establecer parámetros a analizar considerando la diversidad de dimensiones que atraviesan los problemas y lo complejizan. El concepto de complejidad (Morín, 2004) es central en la perspectiva del abordaje pedagógico por cuanto las tradiciones disciplinares han impuesto un modo cartesiano de fragmentar la realidad en preguntas concretas que, si bien contribuyen a su análisis, muchas veces obvian las interacciones y los emergentes que les son 
inherentes y que las modulan permanentemente. Trascender esa limitación conceptual es parte del enfoque en que se estructura la práctica.

Por otra parte, el diagnóstico constituye una fotografía del contexto acotada al momento en que este se realiza y que es delineado por la multidimensionalidad de enfoques desde donde se intentan decodificar los problemas, aunque es la perspectiva de la complejidad -que incorpora a la dialéctica en la comprensión del diagnóstico como una sucesión dinámica de fotogramas con la que es posible componer la película a través del tiempo- la que exige de una aproximación inicial que constituye una suerte de línea de base o de punto de partida con el que pueda ser comparada la evolución de la intervención, siempre que pueda sostenerse en el tiempo. Siendo que la realidad es cambiante, tanto por las características del contexto como por las relaciones que emergen en el territorio como resultado de la presencia de la universidad en él, esta dinámica también reconfigura el contexto, lo transforma y lo modula de forma permanente, lo que obliga a pensar el diagnóstico como algo recurrente.

La multiplicidad de factores a tener en cuenta en el marco de un diagnóstico es tal que requiere e impone un abordaje interdisciplinar y sistémico (García, 2006) de los problemas, en particular cuando estos están relacionados con situaciones estructurales como las que configuran el territorio en que suelen desarrollarse las prácticas de extensión y las prácticas integrales, y que se caracteriza por un hábitat usualmente contaminado por basurales, con conexiones clandestinas de agua que no suelen cumplir los estándares que la califiquen como potable para el consumo, con viviendas precarias, sin gas ni conexiones seguras de electricidad y donde se asienta una población integrada mayoritariamente por migrantes desempleados o con trabajos informales y mal pagos.

Profundizar en el diagnóstico de los problemas implica caracterizarlos y focalizar las causas que los generan y los modos en que estas se concatenan. Desde las ciencias ambientales (química, física, biología, entre otras disciplinas), los estudios cuantitativos en relación con ciertos indicadores de contaminación de agua, aire y suelo -por ejemplo- son importantes y permiten corroborar supuestos previos que usualmente surgen de inferencias cualitativas que pudieron haberse realizado previamente en los barrios a partir de la observación directa o de la descripción del escenario por los propios vecinas/os. Estos muestreos (exhaustivos o aleatorios) constituyen indicadores que pueden incorporarse a la línea de base y a un monitoreo posterior.

Otros enfoques disciplinares pueden sumarse aportando datos sobre el estado estructural de las viviendas, sobre el modo en que se gestiona y administran los recursos básicos (la seguridad de las conexiones de gas, agua, electricidad, etc.), la recolección de la basura y hasta el modo en que se gestiona a nivel comunitario el hábitat, es decir, los espacios comunes, las calles, plazas y espacios verdes, la vegetación y la fauna, tanto mascotas como otras especies que pueden estar presentes en el barrio y entre las cuales pueden incluirse vectores perjudiciales para la salud; mientras que aquellos aspectos relacionados con los vínculos y los lazos personales pueden relevarse a partir de metodologías propias de la etnografía como las encuestas, las entrevistas y las historias de vida, por citar las más conocidas, pero que pueden integrarse a estudios más sofisticados como los análisis de redes (Kadushing, 2004; Palacio, 2009) que permiten comprender los nexos de una trama 
usualmente invisibilizada de problemas, percepciones e interacciones donde emergen referentes, direccionalidades y flujos en relación con los vínculos y otros tantos aspectos que dan cuenta de la complejidad del entorno. La experiencia de trabajo de cátedras que vinculan algunos de los contenidos de las materias que imparten en el territorio y en particular en el marco de los Centros Comunitarios de Extensión Universitaria de la Universidad Nacional de La Plata (CCEU) dan cuenta de estas problemáticas, interacciones y emergentes.

No obstante, no se trata - bajo esta lógica- de invadir la privacidad de los barrios ni la de sus habitantes, y menos aún de considerarlos como objeto de estudio, todo lo contrario. Bajo la perspectiva de una práctica situada, el diagnóstico adquiere gran relevancia, porque es un punto de partida para el establecimiento de las demandas y prioridades, convirtiéndose en un facilitador de la organización comunitaria, aunque esto es posible en tanto pueda garantizarse la integración con la comunidad, de tal modo que esta encuentre, en la presencia de la universidad en el territorio, un aliado en la construcción de una agenda común para la co-gestión y la transformación del contexto. Diagnosticar, entonces, implica una participación activa -o al menos la aceptación- de la comunidad (Bronfman y Gleizer, 1994) y la formulación de preguntas que conducen a uno de los procesos más enriquecedores de este tipo de prácticas, la problematización.

Problematizar el contexto supone indagar en los problemas y abordarlos desde la perspectiva de la complejidad, profundizando en las causas que los originan, en las interacciones que se generan y en los intereses que emergen en torno a estos y que normalmente constituyen un campo de disputa. La problematización de la realidad constituye entonces un proceso del pensamiento colectivo y crítico en el que se articula la experiencia de los hechos cotidianos (Fabre, 2011) y de lo vivencial, con los marcos teóricos desde donde es posible formular las interrogantes que permiten avanzar en su comprensión.

Problematizar no es identificar los problemas, sino explicarlos y, para ello, es preciso establecer los nexos que los articulan, su historicidad y el modo en que han ido evolucionando, precisando los factores que los condicionan, los actores involucrados y los alcances de este involucramiento en la dinámica de transformación. Problematizar el contexto a partir de la profundización del diagnóstico permite, por una parte, elaborar las argumentaciones que avalan la presencia de la universidad en el territorio y establecer, en consecuencia y a partir de las preguntas que surgen de esa problematización, los propósitos de la intervención, estableciendo prioridades.

\section{Justificación y Fundamentación como argumentos de la intervención}

Justificar la presencia de la universidad en el territorio pareciera ser una obviedad. La extensión ha contribuido a conectar a la comunidad académica con la comunidad que se extiende más allá de los claustros y este propósito ha vertebrado el trabajo territorial de las universidades y ha contribuido a marcar, a lo largo de su historia, una impronta que se ha inscripto en las tradiciones académicas. El formato de las prácticas integrales constituye una razón más que suficiente para validar la presencia de la universidad en los barrios, tanto por la relevancia que esta articulación reviste para la sociedad en la contribución que pueden hacer las/os universitarios a la resolución 
de algunas problemáticas, como por la importancia que tienen las prácticas contextualizadas para el aprendizaje y la formación de los futuros profesionales y, en consecuencia, para la sociedad en su conjunto.

Los modos de justificar la presencia de las universidades en el territorio pueden ser variados. Una de estas perspectivas, entendida como tecnocrática, apela a la contribución que para la comunidad representaría el aporte del saber académico en la solución a las problemáticas cotidianas. Esta visión, que se presenta como puramente instrumentalista, racional y despolitizada, encierra, no obstante, una ideología de lo apolítico que no deja de ser política.

Otra de las formas de justificar la intervención de las/os universitarios y el vínculo con la comunidad está asociada a lo hermenéutico interpretativo (Ruedas Marrero et al., 2009; Babich, 2017) y, desde esa perspectiva, se apela a reconstruir las relaciones y los procesos que acontecen en el territorio entendiéndolo como un escenario mediado por una diversidad de interpretaciones que ponen de manifiesto las tensiones, disidencias y los acuerdos que puedan existir entre los diferentes actores que lo habitan, y que pueden ser desencadenantes de conflictos, alianzas o convergencias. Una de las falacias en las que se suele incurrir desde algunas bibliotecas es la de considerar al conflicto como resultante de las relaciones interpersonales y no como procesos sociales que encierran intereses contrapuestos (Entelman, 2002). En las antípodas, una perspectiva democrática y problematizadora entiende al conflicto de intereses como natural, reconociendo en las contradicciones que esos intereses - muchas veces antagónicos- develan potencialidades para la transformación. Los argumentos que justifican, entonces, el rol de las universidades en el territorio, interviniendo en la problematización del contexto en que ocurre esa intervención, son inevitablemente ideológicos.

Si se trata de fundamentar, en cambio, los argumentos, aun cuando guardan cierto nexo con las perspectivas que justifican el trabajo en el territorio, se centran en aportar los marcos teóricos y las metodologías desde donde es concebida la articulación con la comunidad y sus actores. Tal como es posible de inferir, la justificación apela a la relevancia de la intervención de la universidad en el territorio, mientras que es la pertinencia la categoría que permite fundamentarla $y$, en ese sentido, emergen modelos o paradigmas diferentes desde donde es posible construir los vínculos.

El modelo transferencista (Tommasino y Cano, 2016) suele conectar las intenciones y acciones que enmarcan una participación de tipo tecnocrática y algunas formas compatibles con una perspectiva interpretativa desde donde se acompañan los procesos, centrándose fundamentalmente en las individualidades. Desde un paradigma crítico problematizador (Zavaro, 2020b) que es propio de concepciones que conciben a la intervención como un proceso de construcción colectiva de sentido, lo dialógico aparece como un descriptor del vínculo que opera a través de la circulación de la palabra, lo que permite reconocer, en ese contexto, además de problemas concretos - que constituyen el objeto de la intervención-, la centralidad que ejercen las relaciones entre los sujetos, entendiendo que esa relación implica el reconocimiento del otro como un par con quien es posible establecer acuerdos y planificar modos de construir sentido. 
Es la problematización del contexto sobre la base del diagnóstico participativo (Valderrama, 2013), en el marco de un modelo constructivista, democrático, dialógico y crítico, la garantía para conseguir, con la participación activa de la comunidad, transformar lo instituido. Las prácticas integrales entonces aparecen como un formato capaz de fomentar, a partir del diálogo entre saberes diversos, la construcción de una intersubjetividad desde donde no solo es posible la transformación del contexto, sino también la de aquellos actores que en él conviven e intervienen, repensando esa intervención desde una concepción que le asigna a la hipótesis de transformación de la realidad un rol central en el que se aglutinan las voluntades y se formulan los propósitos del trabajo territorial.

\section{El propósito de transformar}

Tal como anticipan los párrafos anteriores, las prácticas integrales persiguen, en principio, dos propósitos: uno de ellos relacionado con la formación del estudiantado en un modo de aprendizaje que les permita integrar contenidos y competencias propias de diversas funciones académicas como la docencia, la investigación, la gestión o la extensión en el marco de un aprendizaje situado y contextualizado, mientras que el otro de los propósitos permitiría contribuir a la transformación del contexto en que se objetivan esas prácticas.

La transformación real no es posible si los objetivos que estructuran el formato no están delineados sobre la base a un diagnóstico certero y problematizado con participación de la comunidad, porque la medida en la que esta se involucra constituye una apuesta a la posibilidad tangible de concretar la transformación. Los marcos teóricos y los argumentos que justifican la intervención, por otra parte, son los que permiten respaldar el proceso, en tanto no solo entiende al contexto como objeto de transformación, sino también a los sujetos que lo habitan.

Esta hipótesis subyacente es aportada por Fals Borda $(1981,1987)$ como horizonte del formato pedagógico: la acción colectiva, además de transformar la realidad, transforma a quienes la transforman en sujetos sentipensantes (Fals Borda, 2009). Es sobre la base de esta premisa que es posible formular el propósito que integra y da sentido al trabajo territorial, conjugando las necesidades de la comunidad en la formulación de la demanda en tanto esta se oriente a responder las preguntas que emergen de la problematización del diagnóstico en la lectura del entorno.

Identificar problemas específicos permitiría delinear no solo objetivos concretos que faciliten la planificación de las acciones a desarrollar, sino también establecer metas como horizonte de esas acciones. En la medida en que esas metas puedan concretarse, se habrán alcanzado los objetivos propuestos como eje de la articulación, para lo cual resulta central definir indicadores en torno a los cuales pueda ser evaluado, de manera sostenida, el desarrollo del proceso. Estos indicadores pueden ser cualitativos o cuantitativos y su elección es crucial si se pretende evaluar, de manera certera, las metas propuestas. 


\section{La metodología como recurso...}

Los objetivos propuestos y las metas que deberían alcanzarse definen, en cierta medida, la metodología de trabajo, que no obstante responde a los marcos teóricos desde donde se concibe la práctica. En principio, es muy difícil que la metodología pueda descontextualizarse del paradigma al que se adscribe y desde el cual se concibe y diseña el espacio, ya que son los constructos y los supuestos que subyacen en las bibliotecas a las que adhieren las/os académicas/os las que, en cierta medida, terminan condicionando las actividades y dinámicas que las articulan. En los equipos heterogéneos, consensuar una metodología resulta algo más complejo, pero muchas veces la dinámica propia que se va generando en la construcción de sentido hacia el interior de estos, y que es mediada por el vínculo que se genera con los miembros de la comunidad que comienzan a involucrarse y por los propios estudiantes, van modulando el espacio y, de alguna manera, lo va constriñendo en términos pedagógicos -y por lo tanto didácticos- a uno u otro formato.

Bajo un modelo transferencista, hay poco espacio para la incorporación de ideas alternativas y de sugerencias y esto, de cierta manera, termina visibilizándose en los modos de relación entre quienes participan, que usualmente terminan por asumir que existe un saber concentrado en unos pocos referentes que son los encargados de transmitir información que, a su vez, tiene un único destino: ser difunda entre el resto de los actores del territorio, siendo esa intervención usualmente limitada a la reproducción acrítica del mensaje, a la aclaración de dudas o a la implementación de acciones que son delineadas por aquellos que se arrogan el monopolio de la palabra y de quienes se esperan indicaciones por ser identificados como autoridades dentro del espacio.

En el marco de un modelo constructivista y dialógico, en cambio, las/os docentes no suelen ejercer un rol de dominación, sino que constituyen articuladores o facilitadores de una construcción colectiva de sentido que se basa en la circulación de la palabra como premisa y bajo la cual el diálogo de saberes, que suele tener un origen diverso, aparece como un proceso reflexivo que es condicionado por la participación y la formulación de ideas sin que se renuncie a la teoría ni al saber académico, sino amalgamándolo con aquellos conocimientos que lo interpelan desde las diversas racionalidades y cosmovisiones que afloran en ese proceso (Leff, 2003).

Esta perspectiva dialógica no solo convierte al espacio en algo dinámico capaz de transformar a quienes participan, sino que esa misma horizontalidad, que caracteriza al modo en que docentes, investigadores, estudiantes, vecinas/os y otros actores se relacionan, fomenta el desarrollo de vínculos personales que, en cierta medida, favorecen el trabajo, promueven el interés común y las relaciones de pertenencia que comienzan a delinearse y permiten elaborar una agenda común, más allá de que esta implique una negociación permanente de intereses.

Entre ambos marcos teóricos, existe un gradiente de posibilidades que complejizan el proceso y lo despojan de posibles apreciaciones reduccionistas. En ambos, la complejidad es inmanente, aun cuando operen diversas lógicas. No obstante, configuraciones cercanas al constructivismo, si bien pudieran pensarse - por su horizontalidad-como una metáfora de la torre 
de Babel, terminan por fomentar propósitos comunes como camino hacia la transformación real del entorno y a un tipo de aprendizaje reflexivo y contextualizado.

La metodología, en síntesis, por su nexo con los marcos teóricos y los propósitos del formato, define y caracteriza al modo en que se configura el espacio, consolidando una suerte de arquitectura con diversos niveles de jerarquía (Figura 2) que puede incluir programas y proyectos (pedagógicamente atados a la currícula) donde conviven dispositivos particulares en los que anidan las actividades que constituyen los instrumentos de mediación en la consecución de los objetivos y que guardan una correlación directa con las expectativas que esos objetivos generan en relación con las metas que se pretenden alcanzar.

\section{El dispositivo como instrumento de la praxis}

Existen diversos imaginarios en torno al término dispositivo (Moro Abadía, 2003) que responden a distintos recortes disciplinares y que comprenden campos de conocimiento diferentes, desde la informática hasta la física o la sociología, aunque, en términos generales -y desde una perspectiva estructuralista- es posible definirlo como un conjunto heterogéneo de elementos que comprende e involucra instituciones, normativas, proposiciones, herramientas, actividades y discursos, entre otros elementos, y que, en su conjunto, terminan por conformar mecanismos en los que estos se articulan y entraman a diferentes niveles y jerarquías, con el fin de abordar una problemática en particular.

\section{Figura 2}

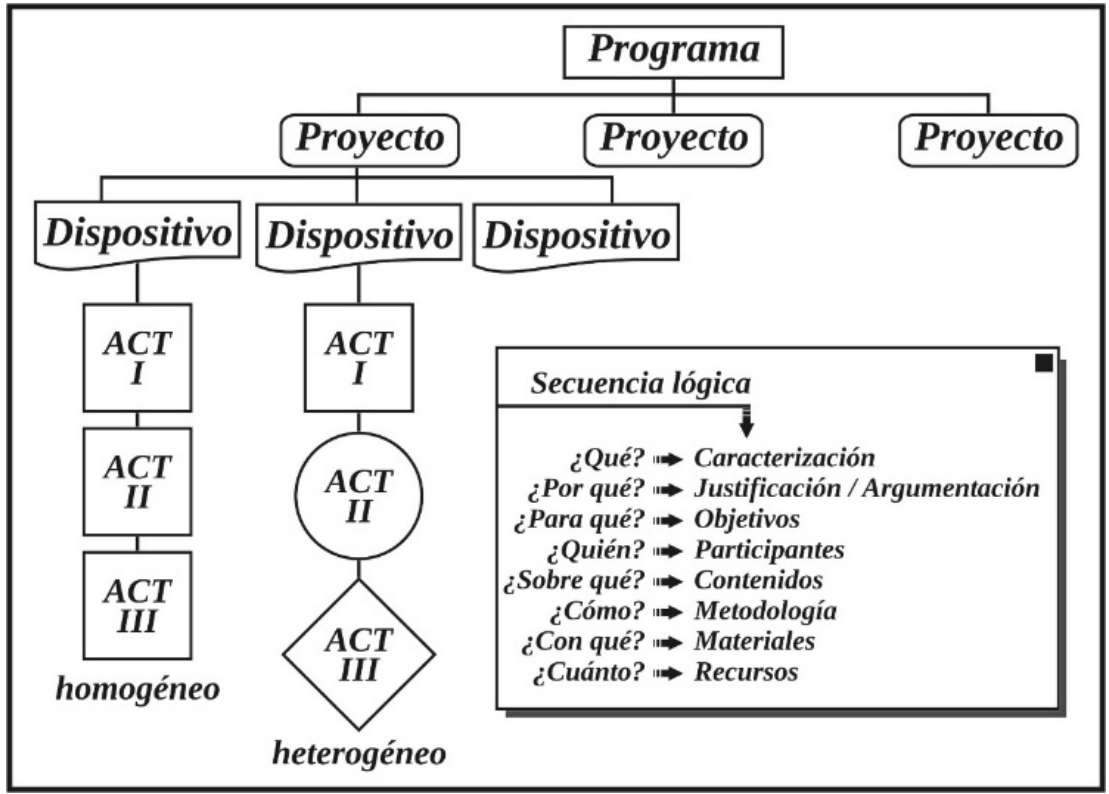

Estructura de un dispositivo en el marco de las prácticas integrales. Secuencia lógica en el diseño de actividades 
En el marco de un proyecto pedagógico como las prácticas integrales, y en relación con el rol que, en este, desempeña la extensión universitaria vinculando a vecinas/os y al contexto en el que es posible enmarcar y objetivar la práctica, el concepto de dispositivo delimitaría una serie de configuraciones particulares que, en función de ciertos objetivos, permitiría articular saberes, actores, disciplinas y prácticas en las que se inscriben actividades diversas tales como talleres, juegos de roles, charlas participativas, ateneos, espacios de reflexión, etc.

Más allá de las jerarquías y del lugar que puedan ocupar cada una en un dispositivo, las actividades constituyen herramientas eficaces que facilitan la objetivación de la práctica. Elegir una u otra define al dispositivo, lo caracteriza e incluso lo delimita. Según sus características, estos dispositivos pueden ser homogéneos o heterogéneos, focalizados u holísticos, efímeros o sostenibles, etc. Estas particularidades responden a diversos factores entre los que se encuentran las especificidades académicas de la propuesta de intervención, la demanda y la posibilidad real de dar respuesta a esta, así como el interés de la comunidad en involucrarse.

Son las actividades las que, en gran medida, condicionan la diversidad de los dispositivos incluso hacia el interior de los mismos. En los dispositivos homogéneos, las actividades que lo estructuran suelen ser similares en forma, dinámica y propósito, mientras que, en aquellos heterogéneos, la diversidad es notoria y en ellos suelen combinarse actividades de diversa naturaleza, pero que en su articulación encuentra sentido el dispositivo, en la medida en que estas van integrándose en respuesta al objetivo general y a las etapas del proyecto o del programa de intervención en los que estos se inscriben.

En el diseño de las actividades, si bien se pretende que respondan al objetivo general, es imprescindible tener en cuenta los objetivos particulares y las metas que a través de ellos se persiguen alcanzar, tanto en relación a los aspectos pedagógicos como a las demandas del territorio y a los interlocutores que conforman el espacio. Son los objetivos específicos los que condicionan la elección de una u otra actividad y su ubicación en la secuencia que conforma el dispositivo.

Las actividades de presentación suelen inaugurar los espacios de trabajo y están orientadas a generar un acercamiento entre las/os participantes, a compartir una síntesis de sus trayectorias o a formular expectativas respecto de aquello que se pretende lograr con el desarrollo del encuentro. Las actividades de diagnóstico, por su parte, están encaminadas a profundizar sobre un problema determinado y suelen ser mediadas por métodos propios de una disciplina en particular o por la conjunción de diversas técnicas y dinámicas, pudiendo utilizarse tanto herramientas cuantitativas como cualitativas. Por su parte, las actividades relacionadas con la problematización están orientadas a profundizar en los diagnósticos previos, a conocer la opinión de la comunidad respecto de estos o a consolidar puntos de vista particulares, mientras que la configuración de un dispositivo puede complementarse con otro tipo de actividades de síntesis como aquellas encaminadas a la reflexión, a la discusión de un tema o de un problema en particular o al intercambio de conocimientos y de saberes de diferente naturaleza y origen, e incluso a la 
divulgación de una temática en particular o de las acciones desarrolladas en el marco de ese espacio.

Si bien los temas que pueden abordarse pueden ser variados, en los colectivos homogéneos, estos suelen circunscribirse a demandas específicas que usualmente están atadas a intereses focalizados propios de su singularidad o a las problemáticas que los atraviesa y aúna, mientras que, en colectivos heterogéneos, los temas suelen ser más generales, siendo uno de los más convocantes las problemáticas socioambientales, que no solo resultan de interés para todas /os, sino que suelen ser recurrentes por el impacto que tienen en la cotidianidad del barrio. La temática ambiental, por su complejidad, suele ser, entonces, un eje organizador del trabajo territorial y en particular de este tipo de prácticas por requerir, en su abordaje, de la integración de disciplinas, de actores y de metodologías diversas (Zavaro, 2020a) y por el hecho de que, al representar un interés común, no solo contribuye a afianzar las relaciones interpersonales, sino también a promover la transformación de esa realidad que les convoca.

Más allá del tema específico que se aborde en cada una de las actividades que conforman un dispositivo en particular, no es casual que, en el diseño de las dinámicas, se privilegien tanto las características de los colectivos con los que se pretende trabajar como las edades y los intereses que los atraviesan. Los colectivos más comunes con los que se suele interactuar en el marco de este tipo de formatos involucra a alumnos de escuelas radicadas en la zona (reforzando temas curriculares de manera contextualizada) o a vecinas/os que o bien están sesgados por ciertas particularidades como es el género (colectivos de mujeres, por ejemplo), la edad (adultos mayores o adolescentes) o los intereses comunes (agrupaciones sociales o políticas) o, por el contrario, son colectivos disímiles, en cuyo caso resulta ser más complicado el diseño de esas actividades por la dificultad que implica generar situaciones que involucren la participación de todos sin que, en medio de estas, decaiga el interés.

Por último, otro aspecto a tener en cuenta en su diseño es el tiempo estimado para desarrollarlas, así como la secuencia de instancias por las que se transita y los materiales que se requieren como intermediación desde el punto de vista didáctico. En esta instancia, resulta central tanto la planificación temporo espacial, que a su vez involucra un cronograma, como los recursos necesarios para su realización. Cronograma y presupuesto, entonces, son elementos claves que definen o limitan la posibilidad de concretar el trabajo.

\section{La planificación situada como garantía de la objetivación}

Planificar el espacio de intervención y en él los dispositivos que lo conforman y las actividades que lo configuran es necesario porque permite ordenar el trabajo con cierta previsibilidad. La planificación supone reconfigurar el modo en que se articulan las experiencias capitalizando la expertiz de las/os docentes-investigadores. En el diseño de la planificación del formato, la gestión, como práctica académica, cobra especial atención si se entiende como una gestión compartida entre la universidad, las instituciones radicadas en el territorio, las/os vecinas/os e incluso las/os estudiantes. Planificar el espacio desde la cogestión como una 
experiencia dinámica y sostenida no solo es garantía de la práctica, que es diseñada colectivamente, resolviendo obstáculos y problemas de forma mancomunada, sino que constituye una práctica y un aprendizaje en sí mismo desde donde desarrollar competencias que aúnan a quienes participan del espacio en torno a un objetivo común que, en principio, está asociado al éxito de esa gestión. Experiencias de trabajo de intervención territorial en el marco de diversas materias que conforman los planes de estudio de carreras de la UNLP y de los contenidos explícitos que conforman sus programas de estudio, pero resignificados por las/os estudiantes en consonancia con el modo de gestión de los CCEU, donde las actividades se conciben y desarrollan a partir de las demandas explícitas de las/os vecinas/os e instituciones, formuladas a través de las mesas barriales, permiten ilustrar este modelo de planificación.

Planificar implica proyectar a futuro sobre la base de objetivos, metas y expectativas delineando etapas que le dan continuidad a la propuesta y se suceden en la medida en que el formato se concreta, consolidando -en su concreción- las metas que alientan los propósitos (objetivos) iniciáticos, ya sean organizativos, en relación con la gestión de la práctica y a la conformación de los equipos de trabajo, en relación con la comunidad y con los modos en que esta se integra a la comunidad académica o en relación con el diseño de los dispositivos que, concebidos como módulos dinámicos, comprenden diversas actividades.

Al planificar el formato, y en consecuencia los dispositivos y las actividades que lo conforman, resulta recomendable apelar a una secuencia de preguntas que -engarzadas- faciliten la lógica del diseño (Figura 2). Planificar, entonces, implicaría responder a estas interrogantes siguiendo un orden preestablecido. La respuesta al ¿qué? permitiría caracterizar la actividad en sentido estricto, es decir, da cuenta de aquellas especificidades que permiten diferenciarlas de otras actividades dentro de un mismo dispositivo o entre dispositivos diferentes, aun cuando puedan constituir un nexo entre estos. El ¿por qué?, por su parte, nos permite conectar la actividad con el propósito de cada uno de los dispositivos y permite reconocer sus fortalezas, siendo conscientes de su importancia como tramo del proceso de construcción de conocimientos y sentidos, mientras que el ¿para qué?garantiza circunscribir la actividad a un objetivo particular que remite a su relevancia, entendiéndolo como nexo con las metas a alcanzar. El ¿quién?, por otra parte, refiere a los sujetos involucrados en el desarrollo de las actividades. Bajo un paradigma transferencista, se circunscribiría a aquellas personas a las que están destinadas las actividades propuestas, mientras que, bajo un modelo constructivista, el quiénes remite al conjunto de actores involucrados, tanto a aquellos que diseñan y planifican las actividades (especialmente si es en el marco de la cogestión) como al resto de los que participan y que, en medio del proceso que conlleva la integración de los actores territoriales, van integrándose a la dinámica de equipo y contribuyen incluso de la construcción de la demanda y a su planificación. El quiénes, en ese proceso, muta desde un ellos, que encarna a la ajenidad, a un nosotros que los integra y contiene en un mismo colectivo.

La temática de cada actividad y, en consecuencia, en el conjunto de aquellas que conforman los dispositivos y que los conectan con otras actividades, en el marco de la dinámica de interacción con el territorio, constituyen el eje que vertebra el ¿sobre qué?, es decir, el conjunto de saberes que 
estructuran conceptualmente la intervención y que operan como una suerte de profundización del enunciado que titula la propuesta, y que puede encontrarse en la respuesta que en torno al ¿qué?, se propone desarrollar desde el proyecto con cada actividad. El ¿cómo?, en cambio, tiene un fuerte componente didáctico que implica detallar el modo en que se ha de desarrollar cada una de las actividades, explicitando las dinámicas y modalidades que la estructuran y que, en relación con las características de los dispositivos en los que se inscriben, con los propósitos originales y con el rango etario de aquellos con quienes se pretende trabajar, define el carácter de las propuestas. El cómo, entonces, incluye las instancias procedimentales y debería contemplar tanto los tiempos estimados para su desarrollo como la elección de materiales adecuados para poder concretarla.

En este sentido, no hay dudas de que los materiales cumplen un rol determinante como mediación entre los saberes en tensión y el propósito de cada actividad. Su elección es fundamental $y$, en muchas ocasiones, dependen tanto de la naturaleza de la actividad como de los contenidos que se intentan abordar, de los recursos existentes $y$, fundamentalmente, de la edad de los participantes. Según la actividad de que se trate, la especificidad de los materiales resulta un punto neurálgico. A modo de ejemplo, resultaría imposible corroborar la existencia de sustancias contaminantes en el agua que se consume en un barrio sin los reactivos adecuados, por lo que contar con ellos resulta imprescindible para lograr el objetivo de la misma. No obstante, en aquellas actividades que involucran lo lúdico o lo reflexivo, lo más importante es apelar a la creatividad en tanto esta constituye una cualidad primordial, sobre todo cuando el presupuesto es acotado.

Usualmente, la elección de una dinámica que se ajuste a la cantidad de participantes, al rango etario o a sus intereses es más relevante que los materiales en sí mismos, y a menudo algunos elementos comunes como un pizarrón, papel afiche, fibrones, tarjetas, una soga o papel y lápiz suelen ser suficientes para poner en común cualquier tema, aunque, en algunas edades y contextos, poder contar con un material gráfico que además sea atractivo y sugerente puede convertirse en un recurso excelente.

Entre los aprendizajes más relevantes dentro de un espacio de formación de estas características, es el diseño y la elección de las dinámicas y de los materiales a utilizar uno de los aspectos que cobra especial trascendencia para las/os estudiantes, porque ellos, al asumir el rol de facilitadores, tienen la oportunidad de poner en juego los saberes aprendidos con anterioridad -incluso en las cursadas convencionales previas-, de integrar modalidades que son propias de las prácticas extensionistas a una planificación en la que los contenidos disciplinares no solo son resignificados, sino también deben ser pensados para un contexto de aplicación que -por la propia particularidad del espacio- está centrado en una mirada dialógica, que no está exenta de imponderables y emergentes. Esta mirada de lo complejo, en la planificación del espacio y de los recursos que se utilizan como mediación, constituye todo un desafío que requiere de microdecisiones y decisiones coyunturales que, no obstante, no suelen quedar por fuera de las macrodecisiones con las que se construye metodológicamente el espacio de trabajo (Edelstein et al., 2012) ni de los marcos pedagógicos que definen las estrategias didácticas que lo configuran. 
Articular el diseño y la planificación del espacio de manera conducente implica, entonces, imaginar una situación puntual -contexto-y delinear, teniendo en cuenta esas circunstancias, una estructura basada en acciones y pautas (Matus, 1987) que son ordenadas cronológicamente de manera intencional y plasmadas en un cronograma desde donde es posible concebir la mejor estrategia de intervención. El cronograma, entonces, constituye una herramienta central de la planificación que, utilizando como prototipo una matriz de Gantt (Figura 3), permite ordenar en sentido vertical las diferentes actividades que conforman los dispositivos en relación con las etapas, pero en un momento determinado (día, semana, mes, etc.), lo que da cuenta de la sincronía de las diversas instancias que caracterizan el formato y que incluso puede comprender actividades propias de un dispositivo en etapas diferentes y a lo largo de los años en que se diseña el programa en relación con el plan de estudios. La lectura del cronograma en sentido horizontal, por su parte, permite visibilizar la regularidad con la que cada una de las actividades se concibe a lo largo del tiempo y, por tanto, de su sostenibilidad.

\section{Figura 3}

\section{Ejemplo del diseño de un cronograma utilizando una matriz de Gantt}

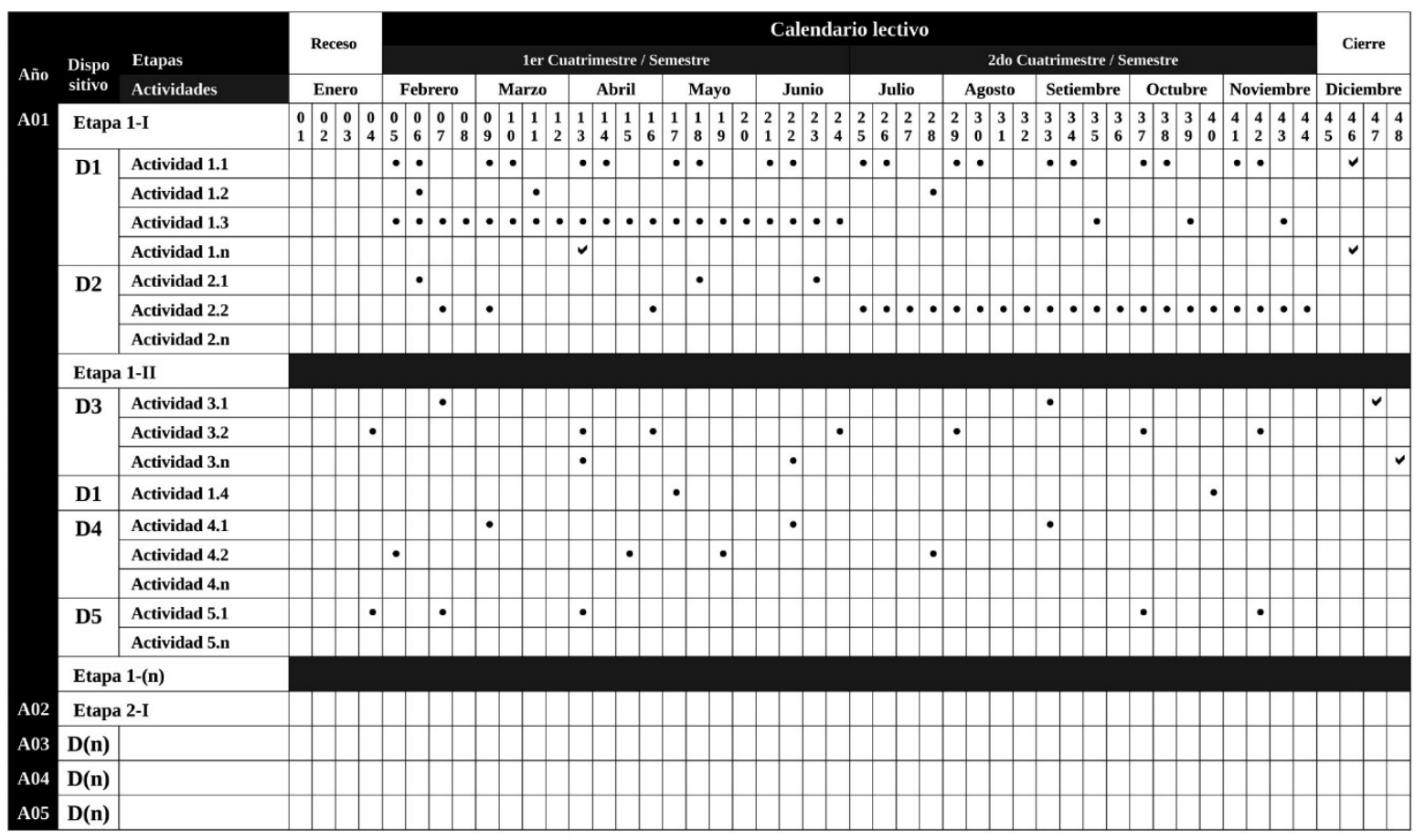

Para que esta planificación constituya un diseño situado, contextualizado y eficiente, es importante considerar tanto los tiempos del territorio, en función de sus necesidades, como las expectativas previstas en los planes de estudio en los que el formato se inscribe, de modo que pueda ajustarse al calendario académico minimizando posibles incompatibilidades que pudieran representar, a priori, una contradicción. Contemplar esta situación en la planificación del formato es importante por la importancia que reviste su curricularización en los planes de estudio y por el 
impacto que esa curricularización tendría en la formación de las/os futuros egresadas/os y en la consolidación del perfil de profesional que es pensado desde la institución. Esa articulación puede lograrse mediante una disociación parcial de las exigencias que el espacio representa para las/os estudiantes, respecto de las metas planteadas para la intervención en relación con la presencia de la universidad en el territorio, que usualmente requiere de prolongar los tiempos de permanencia.

La permanencia sostenida, que es central para cumplimentar el segundo de los propósitos y además para garantizar el respaldo de la comunidad y su credibilidad en el espacio, puede lograrse con proyectos transversales que atraviesen la currícula de tal forma que en el espacio puedan converger materias y estudiantes de años diferentes que lo van transitando en diversas etapas durante toda su carrera o durante gran parte de esta. Ese vínculo sostenido les permitiría formarse paulatinamente en contenidos, experiencias y en la toma de decisiones, pero, a la vez, les posibilitaría consolidar los vínculos con las/os vecinas/os e inaugurar nuevos vínculos con los ingresantes, que podrían comenzar a formarse, tempranamente, de y con quienes son sus pares y tienen una trayectoria acumulada en el trabajo territorial.

La transversalidad permite, además, abordar los problemas en el tiempo, acompañando su evolución y dinámica, así como las decisiones y las demandas de la comunidad, lo que permite profundizar el conocimiento del territorio y fomentar el compromiso con sus problemáticas y con la complejidad que estas representan. Las metas, entonces, si bien se plantean como horizontes y utopías que muchas veces no llegan a concretarse acorde a una planificación estricta, constituyen un punto de acuerdo que permite orientar el trabajo con la comunidad. Por otra parte, gran parte del éxito del formato radica en el clima de trabajo que pueda establecerse. Si las metas pueden ser entendidas como relativas, en un formato de estas características, entonces la evaluación adquiere especial relevancia, no como instancia final del trabajo ni como culminación de la actividad para la acreditación del recorrido de las/os estudiantes por el espacio con impacto en sus trayectorias desde el punto de vista de la calificación, sino como una forma de pensar y de resignificar permanentemente el formato a partir de todo aquello que en él acontece y lo transforma.

\section{La evaluación como actividad...}

Evaluar es una parte fundamental de la actividad académica $y$, en sentido general, constituye una manera de conocer y de analizar el modo en que se trabaja en un campo determinado. La evaluación, en relación con la docencia, ha sido tradicionalmente concebida como una manera de certificar la adquisición de determinados contenidos que son considerados vertebrales dentro del diseño del plan de estudios y, en particular, de los programas de una materia. A menudo, es el/la docente quien evalúa el desempeño de sus estudiantes utilizando como criterio la adquisición de saberes conceptuales sobre la base a sus conocimientos sobre el tema (de Camilloni et al., 1998) y ejerciendo la autoridad que ese conocimiento le otorga.

En el ámbito de las ciencias, son los pares quienes evalúan el trabajo académico (Kreimer 2011) a través de concursos, que constituyen tanto la puerta de acceso a ciertos escaños en la carrera científica -en función de los antecedentes acumulados- como la posibilidad de validar las 
propuestas y los proyectos de investigación a desarrollarse en relación con la importancia que tienen ciertos temas dentro de un campo disciplinar determinado. Este tipo de evaluación es emergente de un modo de producción de conocimientos de tipo endogámico que hoy es interpelado por un modelo de ciencia posacadémica (Jiménez-Buedo y Vielba, 2009) que adquiere cada vez más trascendencia y en el que la sociedad juega un papel importante, tanto en la construcción de la demanda como en el establecimiento indirecto de prioridades que, en términos institucionales, terminan traduciéndose en la jerarquización de algunas líneas de financiamiento.

De igual modo, la extensión también ha implementado mecanismos de evaluación para otorgar, en relación con las propuestas de articulación con la comunidad, el financiamiento que permite que uno u otro proyecto pueda llevarse a cabo, aun cuando usualmente suelen ser evaluados también por los pares, en relación con los antecedentes del equipo y a la coherencia del proyecto, pero donde no suelen incluirse a los miembros de la comunidad como evaluadores de la pertinencia y de la relevancia que este tipo de propuestas podrían tener para la comunidad (Bonicatto et al., 2017). La evaluación, en muchos de estos casos, es erróneamente entendida como sinónimo de acreditación.

No obstante, a diferencia de la acreditación, que consiste en aportar credenciales que permiten certificar ciertas prácticas o legitimar contenidos bajo un formato estandarizado, la evaluación constituye una instancia que posibilita ponderar errores y aciertos, analizar procesos y arribar a conclusiones (Araujo, 2016) que puedan ser relevantes como insumo para modificar la práctica misma cuando esta es entendida desde un punto de vista crítico. En este sentido, una evaluación de tipo procesual, incorporada al formato de las prácticas integrales, permite establecer un seguimiento sostenido en relación con la intervención de la universidad en el territorio y a su articulación con la comunidad, analizando los aspectos positivos y negativos, así como las fortalezas y debilidades emergentes, siendo este proceso relevante como posibilidad de adecuación de la propia intervención a la dinámica que opera en el territorio.

En este aspecto, las metas y los indicadores constituyen referencias que permiten analizar la calidad y sostenibilidad del proyecto y del formato mismo. Siendo además que el espacio, en su concepción, también apunta a la formación de las/os estudiantes bajo la perspectiva de un aprendizaje situado y contextualizado que permite resignificar el saber, trascendiendo los límites del aprendizaje memorístico y de la rutina de los instrumentos evaluatorios tradicionales, la evaluación cobra un sentido de relación que, en términos individuales y colectivos, pondera otros modos de apropiación del saber que involucra a la creatividad, al compromiso y a la responsabilidad. La evaluación, en estos términos, se escinde definitivamente de la acreditación y, lejos de acotarse a una calificación atada a una escala numérica, valora especialmente los análisis cualitativos de las experiencias y los desempeños de los participantes en relación a esta, adquiriendo - por añadidura- un potencial enorme por contribuir a fomentar los vínculos solidarios, el compañerismo y la cohesión de los equipos.

Finalmente, y en relación con la intervención de la universidad en el territorio, esta modalidad de evaluación permite también fortalecer y actualizar el diagnóstico sobre las 
problemáticas y las demandas, analizar el avance y evolución de las mismas y construir sentido, siendo que, en esa construcción, también es posible resignificar tanto los saberes aportados por la comunidad como los propósitos del trabajo comunitario. La mirada cualitativa y sobretodo la opinión de la comunidad en la evaluación de ese formato académico, por otra parte, es novedoso y revolucionario, porque fomenta la perspectiva crítica, la integración de sujetos y el diálogo de saberes que emergen de esa relación, lo que contribuye a resignificar el hacer desde el saber y a retroalimentar el saber desde el hacer, consolidando -en ese proceso- el formato mismo.

\section{Conclusiones}

A modo de conclusión, las prácticas integrales constituyen un espacio con diferentes expectativas e intereses. Contribuyen con la formación de las/os estudiantes desde una práctica situada y contextualizada que les permita desarrollar habilidades, competencias y empatía con los problemas del territorio, interpelando en ese aprender - desde el hacer y el saber- los contenidos que conforman las bibliotecas académicas, e integrando disciplinas y saberes de otra naturaleza entre los que cobran especial importancia los saberes experienciales. Integrar también a otros miembros de la comunidad académica (profesores, investigadores, no docentes y extensionistas) con las/os vecinas/os e instituciones radicadas en el territorio y, en ese vínculo, contribuir a abordar las problemáticas que lo atraviesan constituye uno de los aspectos más relevantes del formato. En el diseño de este tipo de espacios, donde la gestión constituye una garantía del éxito y un aprendizaje más, categorías como contexto, diagnóstico y problematización permiten integrar aspectos de la investigación a tradiciones de la extensión que dan cuenta de la pertinencia y relevancia de esa intervención, fundamentando y justificando la presencia de la universidad en los barrios y planificando, a partir de esas problemáticas, un dispositivo pedagógico capaz de integrar metodologías, actividades, dinámicas y recursos en una propuesta democrática de construcción de conocimientos y de sentido, que permite aprender entre todas/os, transformando -en ese aprender- a quienes participan y a la realidad que les circunda. 


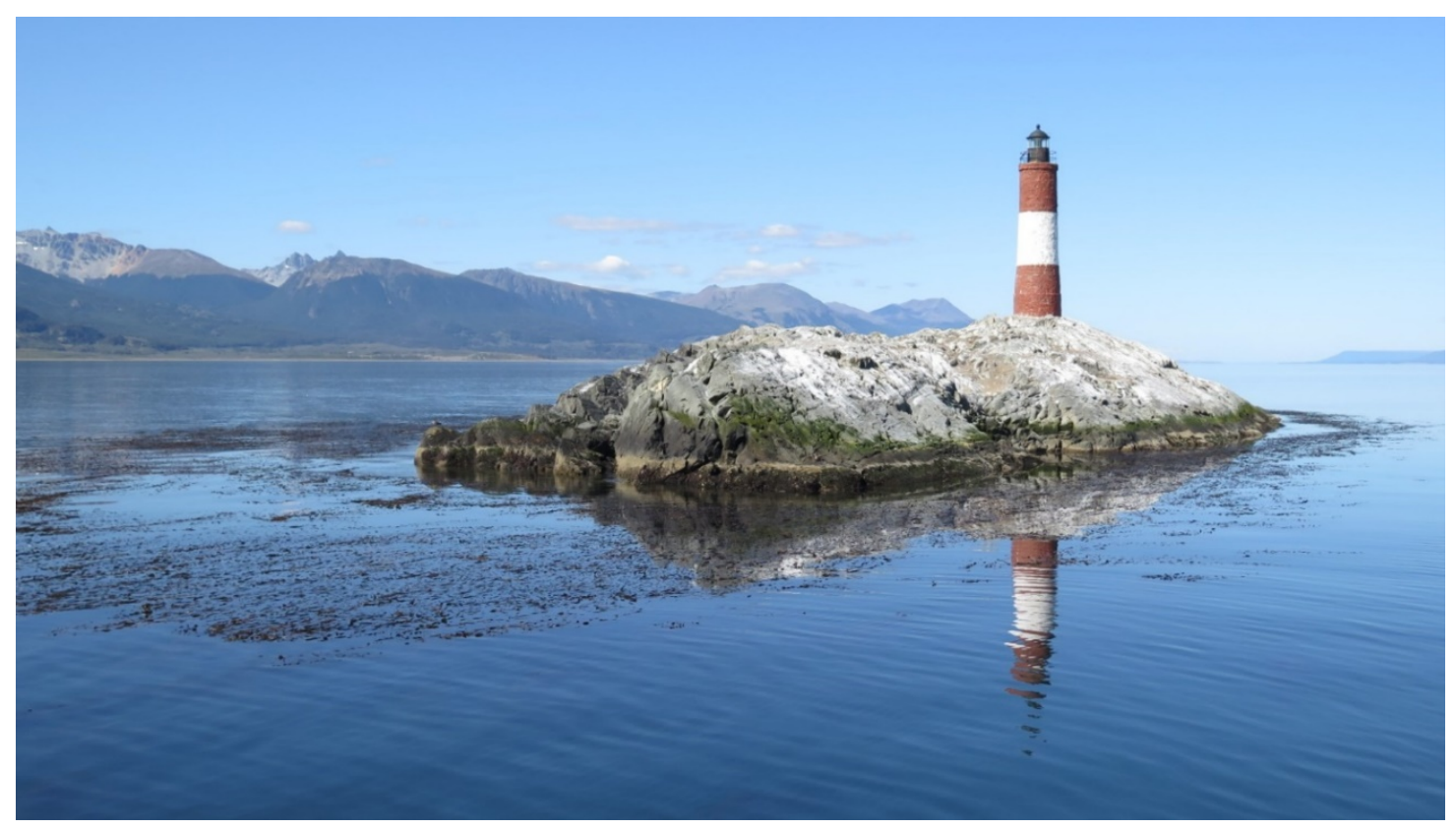

Confin 2, fotografía. Patricia Bonjour

\section{Bibliografía}

Araujo, S. (2016). Tradiciones de enseñanza, enfoques de aprendizaje y evaluación: dos puntos de vista, dos modos de actuación. Trayectorias Universitarias, 2(2), 3-10.

https://revistas.unlp.edu.ar/TrayectoriasUniversitarias/article/view/2753

Ariztía, T. (2017). La teoría de las prácticas sociales: particularidades, posibilidades y límites. Cinta de moebio, 59, 221-234. https://dx.doi.org/10.4067/S0717-554X2017000200221

Babich, B. (2017). Hermeneutic Philosophies of Social Science. Walter de Gruyter GmbH \& Co KG.

Bonicatto, M., Bermúdez, L., Calvo, V., Gómez, M. I. y Moratti Serrichio, F. (2017). ¿Quién te mira y quién te ve?. En Santos, C., Stevenazzi, F., Romero, F., Moratti Serrichio, F., Tommasino, H., Almada, J., Tomatis, K., Bermúdez, L., Carignano, M., Bonicatto, M., Gómez, M. I., González, M.N., Grabino, V. y Calvo, V. (Eds.), Fronteras Universitarias en el MERCOSUR. Debates sobre la evaluación en prácticas en extensión (pp. 29-37). Facultad de Filosofía y Humanidades, Universidad Nacional de Córdoba.

Bronfman, M. y Gleizer, M. (1994). Participación comunitaria: necesidad, excusa o estrategia? O de qué hablamos cuando hablamos de participación comunitaria. Cadernos de saúde pública, 10, 111-122. https://www.scielosp.org/article/csp/1994.v10n1/111-122/es/

Carpinetti, B. (2005). De la expulsión al comanejo. APN.

de Camilloni, A., Celman, S., Litwin, E. y Palou de Maté, M. (1998). La evaluación de los aprendizajes en el debate didáctico contemporáneo. Paidós.

Edelstein, G., Salit, C., Domjan, G., Gabarini, P. y Sívori, L. (2012). Indicios acerca de la construcción de conceptos sobre lo metodológico en la enseñanza. Cuadernos de Educación, 5, 141-157.

Entelman, R. F. (2002). Teoría de conflictos. Gedisa.

Fabre, M. (2011). O que é problematizar? Géneses de um paradigma. Saber \& Educar, 16, 18-29. http://dx.doi.org/10.17346/se.vol16.17

Fals Borda, O. (1981). La Ciencia del Pueblo. En Vio Grossi, F., Gianotten, V., y Wit, T. D. (Eds.), Investigación Participativa y Praxis Rural. Nuevos conceptos en educación y desarrollo comuna/ (pp. 19-47). Mosca Azul. 
Fals Borda, O. (1987). Democracia y participación: algunas reflexiones. Revista Colombiana de Sociología, 5(1), 35-40.

Fals Borda, O. (2009). Una sociología sentipensante para América Latina. Siglo del Hombre Editores.

García, R. (2006). Sistemas complejos. Gedisa.

Giraudo, C. y Vergerio, G. (2017). La extensión universitaria: en busca del saber, del conocimiento colectivo y la construcción de la demanda. Revista EXT,1-19.

http://www.beu.extension.unicen.edu.ar/xmlui/handle/123456789/39

Jiménez-Buedo, M. y Vielba, I. R. (2009). ¿Más allá de la ciencia académica?: Modo 2, ciencia posacadémica y ciencia posnormal. Arbor, 738, 721-737. https://doi.org/10.3989/arbor.2009.738n1048

Kadushin, C. (2004). Introduction to social network theory. Ma.

Kreimer, P. (2011). La evaluación de la actividad científica: desde la indagación sociológica a la burocratización. Dilemas actuales. Propuesta educativa, 36, 59-77. https://www.redalyc.org/pdf/4030/403041707007.pdf Leff, E. (2003). Racionalidad ambiental y diálogo de saberes: sentidos y senderos de un futuro sustentable. Desenvolvimento e meio ambiente, 7,13-40.

http://www.ppgaa.propesp.ufpa.br/pdfs/prosel2020/DAFDS/D3.pdf Matus, C. (1987). Política, planificación y gobierno. Altadir.

Morín, E. (2004). La epistemología de la complejidad. Gazeta de Antropología, 2, 1-14. http://hdl.handle.net/10481/7253

Moro Abadía, ó. (2003). ¿Qué es un dispositivo? Empiria: Revista de Metodología de Ciencias Sociales, 6, 29-46. http://dx.doi.org/10.5944/empiria.6.2003.933

Palacio, D. (2009). Redes de parentesco y entorno natural: apuntes para un diagnóstico ambiental participativo. Trabajo Social, 11,71-86.

Rodrigo, M. J. (1994). Contexto y desarrollo social. Síntesis.

Ruedas Marrero, M., Ríos, M. y Nieves, F. (2009). Hermenéutica: la roca que rompe el espejo. Investigación y Postgrado, 24(2), 181-201. https://dialnet.unirioja.es/servlet/articulo?codigo=3620425

Tommasino, H. y Cano, A. (2016). Modelos de extensión universitaria en las universidades latinoamericanas en el siglo XXI: Tendencias y controversias. Universidades, 67,7-24.

http://www.beu.extension.unicen.edu.ar/xmlui/handle/123456789/99

Valderrama, R. (2013). El diagnóstico participativo con cartografía social: innovaciones en metodología investigación-acción participativa (IAP). Anduli: Revista Andaluza de Ciencias Sociales, 12, 53-65. https://revistascientificas.us.es/index.php/anduli/article/view/3635

van Dijk, T. (2001). Algunos principios de una teoría del contexto. Revista latinoamericana de estudios del discurso, $7(1), 69-82$.

Zavaro, C. (2019). Las prácticas de extensión como experiencias de curricularización: entre el debate y la práctica objetivada. Redes de Extensión, 5, 7-22.

http://revistascientificas.filo.uba.ar/index.php/redes/article/view/6159

Zavaro, C. (2020a). Saberes ambientales y extensión como sustrato de las prácticas integrales. Revista EXT, 12, 1-15. http://revistas.unc.edu.ar/index.php/ext/article/view/30566

Zavaro, C. (2020b). Hacia una taxonomía de las prácticas extensionistas en perspectiva histórica. Universidad en Diálogo: Revista de Extensión, 10(2), 97-124. https://doi.org/10.15359/udre.10-2.5 